\title{
Two-beam dynamical diffraction solution of the phase problem: A determination with $\mathrm{x}$-ray standing-wave fields
}

\author{
M. J. Bedzyk \\ Hamburger Synchrotronstralungslabor (HASYLAB), Deutsches Elektronen-Synchrotron (DESY), \\ D-2000 Hamburg 52, Germany \\ and Cornell High Energy Synchrotron Source (CHESS), School of Applied and Engineering Physics, \\ Cornell University, Ithaca, New York 14853 \\ G. Materlik \\ Hamburger Synchrotronstralungslabor (HASYLAB), Deutsches Elektronen-Synchrotron (DESY), D-2000 Hamburg 52, Germany
}

(Received 6 May 1985)

\begin{abstract}
Using the dynamical theory of Bragg diffraction, we show that there is a direct relationship between the phase of the $x$-ray standing-wave field and the phase of the structure factor. For an experimental demonstration, we monitor the $\mathrm{Ga}$ and $\mathrm{As} K$ fluorescence and resonant Raman scattering yields, while scanning through the (111) and (200) Bragg diffraction rocking curves of GaAs perfect single crystals. The phases of the (111) and (200) GaAs structure factors are determined from the phases of the modulations in these secondary yields. With the use of monochromatized synchrotron radiation, with a variable photon energy between 10 and $15 \mathrm{keV}$, the effect of anomalous dispersion on the structure-factor phases is clearly seen in the vicinity of the Ga and As $K$ absorption edges. We use this feature, in conjunction with the measured absorption spectrum, for directly determining the dispersion parameters $f^{\prime}$ and $f^{\prime \prime}$ for atoms which appear in single-crystal structures.
\end{abstract}

\section{INTRODUCTION}

When an $\mathrm{x}$-ray plane wave is dynamically diffracted ${ }^{1,2}$ by the $(h k l)$ diffraction planes of a thick single crystal, the incident and diffracted plane waves interfere to form an $\mathrm{x}$-ray standing-wave field (XSW). The antinodal planes of this wave field are parallel to and have the same periodicity as the diffraction planes. In the reflection geometry, the relative phase between the standing-wave field and the diffraction planes can be tuned over a range of $\pi$ radians by adjusting the incidence angle in the vicinity of the Bragg angle $\theta_{B}$. For an incidence angle $\theta$ well below the strong Bragg reflection, the standing-wave field is in counterphase with respect to the diffraction planes. As $\theta$ is advanced through the strong reflection, the interference pattern moves in a continuous fashion in the $-\mathbf{H}$ direction. For $\theta$ well above the strong reflection, the antinodes coincide with the Bragg diffraction planes.

Since the photoeffect for inner electrons (in the dipole approximation) is proportional to the $E$-field intensity at the center of an atom, this movement of the standingwave field relative to the diffraction planes can be observed by monitoring the characteristic photoeffect yields from atoms which occupy positions within the periodicity. Batterman ${ }^{3}$ made the first observation of this effect for $\mathrm{x}$ rays by monitoring the $\mathrm{Ge} K$ fluorescence while scanning in angle $\theta$ through the $\mathrm{Ge}(220)$ Bragg reflection. As a further development, Golovchenko et al. used this movement of the standing-wave field for registering the positions of impurities in ${ }^{4}$ and on the surface ${ }^{5}$ of single crystals. More recently, Materlik and Zegenhagen ${ }^{6}$ demonstrated the great advantages of using synchrotron radiation in this field and Bedzyk and Materlik ${ }^{7}$ demonstrated the use of higher-order-harmonic XSW measurements for determining the thermal vibrational amplitude of an adsorbate on a single-crystal surface. In addition, Hertel et al. ${ }^{8}$ showed, that the concept of Fourier transforms can be used to determine important features of the fluorescence-selected atomic distribution function.

As previously stated, the position of the standing-wave antinode between two adjacent diffraction planes has a range corresponding to one-half of a $d$ spacing, and the lower boundary of this range is the $(h k l)$ Bragg diffraction plane. The question that we will presently address is as follows: Where is this boundary or Bragg diffraction plane relative to the unit cell of the structure? From Laue's ${ }^{1}$ derivation for the transparent crystal, it can be shown that this boundary would contain a center of symmetry for a nonabsorbing centrosymmetric structure. In previous discussions ${ }^{2,9}$ on this subject, it was assumed that this symmetry position for the boundary would be preserved for an absorbing centrosymmetric crystal. For noncentrosymmetric reflections, it was realized ${ }^{1,9,10}$ that this boundary would have a nonsymmetric position due to the nonsymmetric elastic scattering distribution. We will show in this analysis, that absorption contributes a general shift to this boundary in the $+\mathbf{H}$ direction. Therefore, this boundary, which we will continue to call the Bragg diffraction plane, occupies a nonsymmetric position for centrosymmetric as well as noncentrosymmetric structures. With this new $\mathrm{x}$-ray standing-wave definition for the Bragg diffraction planes, the phase of the $(h, k, l)$ Bragg diffraction planes relative to the lattice will be shown to be equivalent to the phase of the structure factor $F_{H}$. 


\section{THEORY}

The structure factor, which describes the superposition of the coherent $\mathrm{x}$-ray scattering effects from the $N$ atoms within the unit cell, can be written as

$$
\begin{aligned}
F_{H} & =\left|F_{H}\right| \exp \left(i \beta_{H}\right) \\
& =\sum_{n=1}^{N}\left[f_{n}^{0}(H)+f_{n}^{\prime}(H)+i f_{n}^{\prime \prime}(H)\right] s_{n}(H) D_{n}(H),
\end{aligned}
$$

where $s_{n}(H)=\exp \left(2 \pi i \mathbf{H} \cdot \mathbf{r}_{n}\right)$ is a geometrical phase factor for the $n$th atom and $D_{n}(H)$ is the Debye-Waller temperature factor for the $n$th atom. The atomic form factor $f_{n}^{0}(H)$ accounts for the elastic scattering from the electron distribution of the $n$th atom in the "free electron approximation." The anomalous dispersion parameters $f_{n}^{\prime}(H)$ and $f_{n}^{\prime \prime}(H)$ are added to $f_{n}^{0}(H)$ to describe the influence of absorption processes which lead to incoherent scattering. The complex scattering density function is expressed as ${ }^{1}$

$$
\rho(\mathbf{r})=\sum_{H} \rho_{H}(\mathbf{r})=(1 / V) \sum_{H} F_{H} \exp (-2 \pi i \mathbf{H} \cdot \mathbf{r}),
$$

where the $H$ th component of the scattering density is

$$
\rho_{H}(\mathbf{r})=(1 / V)\left|F_{H}\right| \exp \left[i\left(\beta_{H}-2 \pi \mathbf{H} \cdot \mathbf{r}\right)\right] .
$$

If anomalous dispersion is neglected, $\rho(\mathbf{r})$ becomes the electron density.

It is well established ${ }^{11}$ that measurements of diffracted intensities can directly lead to accurate values for the magnitudes $\left|F_{H}\right|$ of structure factors in crystalline materials. However, due to the geometrical arrangement of the atoms and due to anomalous dispersion, $F_{H}$ is a complex quantity. Therefore, it is also necessary to determine the phases $\beta_{H}$ of the structure factors, if one wishes to directly reconstruct the periodic atom arrangement for an unknown structure (the well-known phase problem of crystallography) or if one wants to determine the

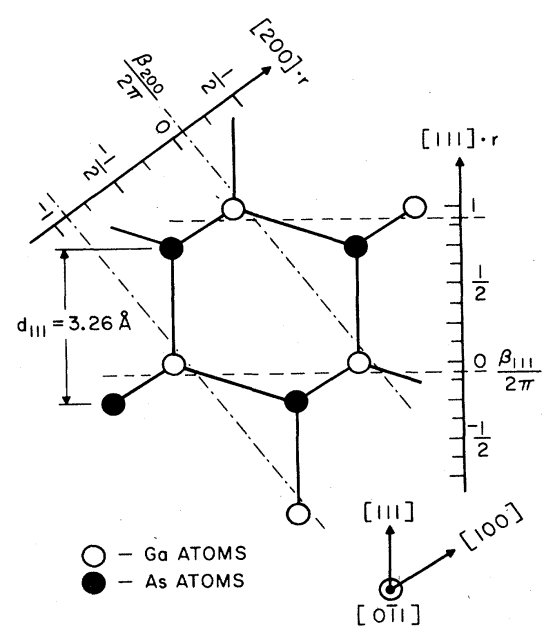

FIG. 1. Schematic view showing the position of the GaAs(111) Bragg diffraction planes (dashed lines) and (200) diffraction planes (dash-dot lines) relative to the $\mathrm{Ga}$ atoms (open circles) and As atoms (closed circles). The absolute position of these diffraction planes corresponds to an energy near the As $K$ edge. (See text for details.) anomalous dispersion parameters in a known structure.

By using the dynamical theory of x-ray diffraction, Laue $^{1}$ showed, in detail for the case of a transparent perfect crystal, that the phase of the structure factor has a direct effect on the phase of a Bragg diffracted $x$-ray plane wave. We will extend this formulation for the phase to include the case of an absorbing crystal. Furthermore, we will show that $\beta_{H}$ values can be measured for any thick perfect crystal by using the $x$-ray standingwave (XSW) technique, ${ }^{3-10,12,13}$ which monitors the phase of the X-ray interference field relative to the phase of a sublattice structure factor containing all atoms of a particular element by recording secondary emission signals (such as fluorescence radiation) from that particular element.

As a demonstration, we will experimentally determine the (111) and the (200) structure factor phases for GaAs. Since the geometrical arrangement of this noncentrosymmetric structure, as shown in Fig. 1, is known to be zincblende, the phase information will be used to determine the anomalous dispersion parameters in the vicinity of the respective $K$ absorption edges.

Though the relative phase between the standing-wave field and the lattice is independent of the choice of the origin of $\mathbf{r}$, the absolute phases of the structure factor and diffracted plane wave are dependent on this choice. We will arbitrarily choose the origin to coincide with a $\mathrm{Ga}$ site and pick the [111] direction to point along a $\mathrm{Ga}-\mathrm{As}$ bond. This places the four $\mathrm{Ga}$ atoms in the unit cell at the fcc positions $\mathbf{r}_{\mathrm{Ga}}^{(1)}=(0,0,0), \quad \mathbf{r}_{\mathrm{Ga}}^{(2)}=\left(\frac{1}{2}, \frac{1}{2}, 0\right), \quad \mathbf{r}_{\mathrm{Ga}}^{(3)}$ $=\left(\frac{1}{2}, 0, \frac{1}{2}\right), \mathbf{r}_{\mathrm{Ga}}^{(4)}=\left(0, \frac{1}{2}, \frac{1}{2}\right)$ and the four As atoms at positions $\mathbf{r}_{\mathrm{As}}^{(i)}=\mathbf{r}_{\mathrm{Ga}}^{(i)}+\left(\frac{1}{4}, \frac{1}{4}, \frac{1}{4}\right), i=1,2,3,4$. The projection of these positions in the [0 $\overline{1} 1]$ plane is illustrated in Fig. 1. With this choice of origin, the geometrical phase factors used in Eq. (1) are $s_{\mathrm{Ga}}(H)=1, s_{\mathrm{As}}(200)=s_{\mathrm{As}}(\overline{2} \overline{0} \overline{0})=-1$, $s_{\mathrm{As}}(111)=-i$, and $s_{\mathrm{As}}(\overline{1} \overline{1})=i$. The position of the $s$ (111) values in the complex plane are illustrated in Fig.

2. The real $\left(F_{H}^{\prime}\right)$ and imaginary $\left(F_{H}^{\prime \prime}\right)$ components for

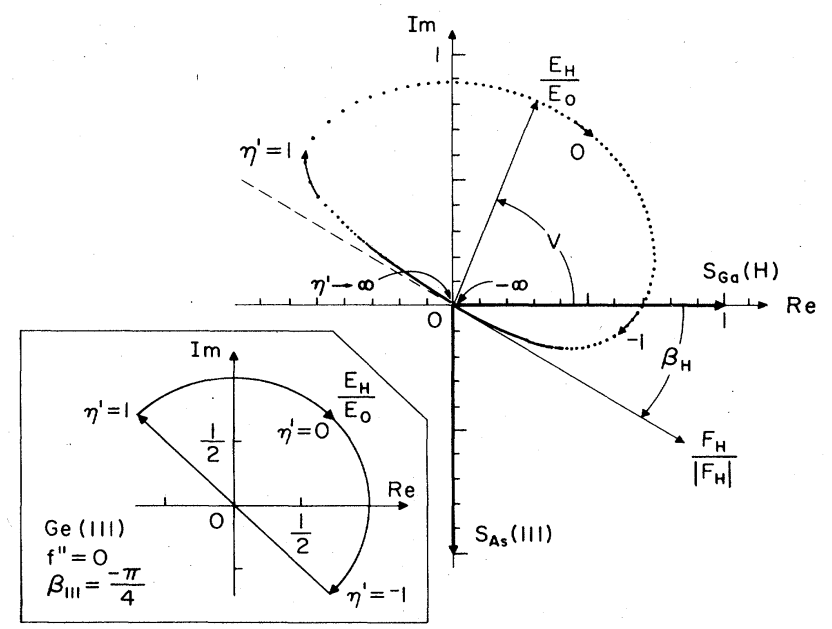

FIG. 2. A complex plane view of the $E$-field amplitude ratio in relation to the structure factor for GaAs(111) at $E_{i}=E_{K}^{\mathrm{As}}-6$ $\mathrm{eV}$. As a comparison, the case for $\mathrm{Ge}(111)$ with no absorption is shown as an inset. (See text for details.) 
$F_{H}=F_{H}^{\prime}+i F_{H}^{\prime \prime}$, corresponding to this origin, can be found in Ref. 9. The phase of the structure factor is defined as $\beta_{H}=\arctan \left(F_{H}^{\prime \prime} / F_{H}^{\prime}\right)$. Referring to Eq. (1) and assuming $D_{\mathrm{As}}=D_{\mathrm{Ga}}$, the phases of the GaAs structure factors used in this demonstration are

$\beta_{ \pm(111)}=\mp \arctan \left(\frac{f_{\mathrm{As}}^{0}(H)+f_{\mathrm{As}}^{\prime}(H) \mp f_{\mathrm{Ga}}^{\prime \prime}(H)}{f_{\mathrm{Ga}}^{0}(H)+f_{\mathrm{Ga}}^{\prime}(H) \pm f_{\mathrm{As}}^{\prime \prime}(H)}\right)$

and

$\beta_{ \pm(200)}=\arctan \left(\frac{f_{\mathrm{Ga}}^{\prime \prime}(H)-f_{\mathrm{As}}^{\prime \prime}(H)}{f_{\mathrm{Ga}}^{0}(H)+f_{\mathrm{Ga}}^{\prime}(H)-f_{\mathrm{As}}^{0}(H)-f_{\mathrm{As}}^{\prime}(H)}\right)$.

The energy dependent $f^{\prime}(0)$ and $f^{\prime \prime}(0)$ dispersion parameter values from Refs. 14-17, which were used in this demonstration for a comparison to our results, are shown as solid and dashed lines in Fig. 3(a). Wagenfeld ${ }^{18}$ has shown, neglecting extended $x$-ray-absorption fine structure (EXAFS) and x-ray-absorption near-edge structure (XANES) spectral features, that in the dipole approximation for the photoeffect, $f^{\prime \prime}(H)=f^{\prime \prime}(0)$, and that deviations from this equivalence should only become significant for transitions from higher orbitals and/or diffraction from higher $(h, k, l)$ indices at higher energies. From Ref. $19 f_{\mathrm{Ga}}^{0}(111)=26.663, f_{\mathrm{As}}^{0}(111)=28.170, f_{\mathrm{Ga}}^{0}(200)$ $=25.778$, and $f_{\mathrm{As}}^{0}(200)=27.168$, and from Ref. 14, the room temperature Debye-Waller factors are $D_{\mathrm{Ga}}(111)$ $=D_{\mathrm{As}}(111)=0.979$ and $D_{\mathrm{Ga}}(200)=D_{\mathrm{As}}(200)=0.972$. The energy dependence of the GaAs(111) and GaAs(200) structure factor phases, obtained by using values from Refs. 14-17 and 19 in Eq. (4), are shown as solid lines in Figs. 3(b) and 3(c), respectively.

Making extensive use of the notation used by Batterman and $\mathrm{Cole}^{2}$ for the two-beam plane-wave case of dynamical x-ray Bragg diffraction, the intensity of the total $E$ field is derived from the sum of the two plane waves as

$$
\begin{aligned}
I= & \mid \mathbf{E}_{0} \exp \left[-i\left(2 \pi \mathbf{K}_{0} \cdot \mathbf{r}-\omega t\right)\right] \\
& +\left.\mathbf{E}_{H} \exp \left[-i\left(2 \pi \mathbf{K}_{H} \cdot \mathbf{r}-\omega t\right)\right]\right|^{2} \\
= & \left|E_{0}\right|^{2}\left[1+\left|\frac{E_{H}}{E_{0}}\right|^{2}+2 P\left|\frac{E_{H}}{E_{0}}\right| \cos (v-2 \pi \mathbf{H} \cdot \mathbf{r})\right] \\
& \times \exp \left(-\mu_{z} z\right) .
\end{aligned}
$$

Here we have used (1) Bragg's law $\mathbf{K}_{H}=\mathbf{K}_{0}+\mathbf{H}$, (2) the polarization constant $P$ which equals 1 for $\sigma$ polarization and $\cos 2 \theta_{B}$ for $\pi$ polarization, (3) the effective attenuation coefficient $\mu_{z}=4 \pi K^{\prime \prime}$, and (4) defined the complex ratio of the $E$-field amplitudes in polar form as

$$
\frac{E_{H}}{E_{0}}=\left|\frac{E_{H}}{E_{0}}\right| \exp (i v) \text {. }
$$

Using the normalized angular parameter ${ }^{2}$

$$
\eta=\eta^{\prime}+i \eta^{\prime \prime}=\frac{b \Delta \theta \sin 2 \theta_{B}+\frac{1}{2} \Gamma F_{0}(1-b)}{\Gamma|P||b|^{1 / 2}\left(F_{H} F_{\bar{H}}\right)^{1 / 2}},
$$

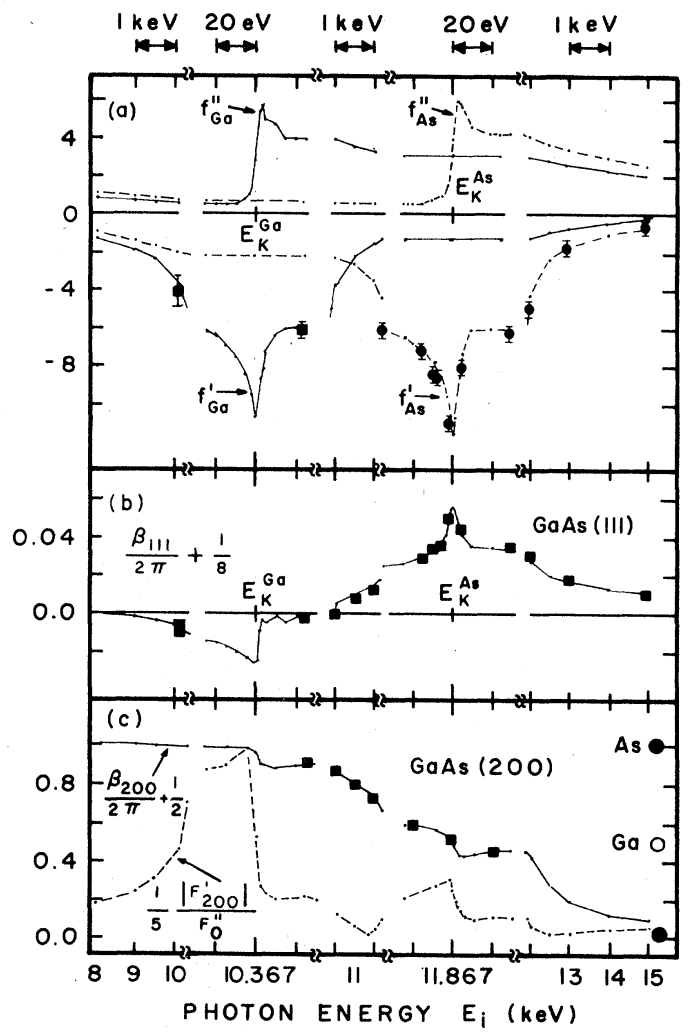

FIG. 3. Energy dependence of (a) the Ga and As anomalous dispersion factors $f^{\prime}$ and $f^{\prime \prime}$, (b) the GaAs(111) structure factor phase $\beta_{111}$, and (c) the GaAs(200) structure factor phase $\beta_{200}$ and reflection strength $\left|F_{200}^{\prime}\right| / F_{0}^{\prime \prime}$. Note that the common energy scale for (a), (b), and (c) has $20-\mathrm{eV}$ increments in the two regions containing the $\mathrm{Ga}$ and $\mathrm{As} K$ edges and $1-\mathrm{keV}$ increments in the three surrounding regions. On the far right side of (c) are shown the positions of the $\mathrm{As}(200)$ and $\mathrm{Ga}(200)$ atom layers with respect to the diffraction plane position at $\beta_{200} / 2 \pi$. The values represented by the solid and dashed lines in (a), (b), and (c) were obtained from Refs. 14-17 and 19 and Eq. (4). The data values shown in (a), (b), and (c) as solid squares or circles were determined from the present study.

$$
\frac{E_{H}}{E_{0}}=-|b|^{1 / 2} \frac{|P|}{P}\left(\frac{F_{H}}{F_{\bar{H}}}\right)^{1 / 2}\left[\eta \pm\left(\eta^{2}-1\right)^{1 / 2}\right] .
$$

For the Bragg reflection geometry, the ratio of the direction cosines $b=\gamma_{0} / \gamma_{H}$ is negative, and has a value $=-1$ when the surface normal is parallel to $\mathbf{H}$. Values for $E_{H} / E_{0}$ are plotted in the complex plane of Fig. 2 for equally incremented values of $\eta^{\prime}$. It is convenient to use the substitution

$$
\eta=\left\{\begin{array}{l}
\cosh (u) \text { for } \eta^{\prime}\left[1+\left(\eta^{\prime \prime} / \eta^{\prime}\right)^{2}\right]^{1 / 2}>1 \quad \text { (case I) } \\
\sin (v) \text { for }\left|\eta^{\prime}\left[1+\left(\eta^{\prime \prime} / \eta^{\prime}\right)^{2}\right]^{1 / 2}\right| \leq 1 \quad \text { (case II) } \\
-\cosh (u) \text { for } \eta^{\prime}\left[1+\left(\eta^{\prime \prime} / \eta^{\prime}\right)^{2}\right]^{1 / 2}<-1
\end{array}\right.
$$

This separation into three cases is to accommodate the $\left(\eta^{2}-1\right)^{1 / 2}$ term in Eq. (8). Case II corresponds to the an- 
gular region of strong reflection. Cases I and III correspond, respectively, to the low- and high-angle side of the strong reflection region. Using the above substitution, Eq. (8) can be transformed into polar form as

$$
\begin{aligned}
\frac{E_{H}}{E_{0}}=|b|^{1 / 2}\left[\frac{\left|F_{H}\right|}{\left|F_{\bar{H}}\right|}\right]^{1 / 2} \exp \left[i \tau \pi+(i / 2)\left(\beta_{H}-\beta_{\bar{H}}\right)\right. & \\
+ & +\left\{\begin{array}{l}
i \pi-u] \text { (case I) } \\
i(v+\pi / 2)] \text { (case II) } \\
-u] \text { (case III), }
\end{array}\right.
\end{aligned}
$$

where $\tau=(1-|P| / P) / 2$. Referring to Eqs. (6) and (10), the magnitude of the ratio of the $E$-field amplitudes is

$$
\left|\frac{E_{H}}{E_{0}}\right|=|b|^{1 / 2}\left(\frac{\left|F_{H}\right|}{\left|F_{\bar{H}}\right|}\right)^{1 / 2} \exp \left(\left\{\begin{array}{l}
\left.-u^{\prime}\right)(\text { cases I and III) } \\
\left.-v^{\prime \prime}\right)(\text { case II) }
\end{array}\right.\right.
$$

and the phase of the amplitude ratio is

$v=\tau \pi+\frac{1}{2}\left(\beta_{H}-\beta_{\bar{H}}\right)+ \begin{cases}-u^{\prime \prime}+\pi \quad \text { (case I) } \\ v^{\prime}+(\pi / 2) \quad \text { (case II) } \\ -u^{\prime \prime} \quad \text { (case III) }\end{cases}$

For the transparent case (i.e., no absorption $f^{\prime \prime}=0$ ), $\left|F_{H}\right|=\left|F_{\bar{H}}\right|, \beta_{H}=-\beta_{\bar{H}}, \eta^{\prime \prime}=u^{\prime \prime}=v^{\prime \prime}=0$.

From the $P \cos (v-2 \pi \mathbf{H} \cdot \mathbf{r})$ factor in Eq. (5), it can be seen that the antinodal planes of the standing wave are (1) perpendicular to the reciprocal lattice vector $H,(2)$ have a spatial period of $d=1 /|H|$, and (3) are displaced in the $\mathbf{H}$ direction from the origin of the unit cell by an amount $\Delta d_{0}=(v / 2 \pi) d$ for $P>0$ and by $\Delta d_{0}=[(v-\pi) / 2 \pi] d$ for $P<0$. The phase of the standing-wave antinode is then related to the phase of the diffracted plane-wave amplitude as

$$
2 \pi\left(\Delta d_{0} / d\right)=v-\tau \pi
$$

The position of the antinode $\Delta d_{0} / d$ as a function of angular parameter $\eta^{\prime}$ is illustrated in Fig. 4. Notice that the asymptotic limits of the antinode position do not coincide with the centers of symmetry (at $-\frac{1}{8}$ and $\frac{3}{8}$ ), but are slightly displaced in the $+\mathbf{H}$ direction. The $\eta^{\prime} \rightarrow-\infty$ asymptotic limit for the antinode position (or Bragg diffraction plane) and the $\eta^{\prime} \rightarrow+\infty$ limit are defined analytically as

$$
\frac{\Delta d_{0}}{d}=\frac{1}{4 \pi}\left(\beta_{H}-\beta_{\bar{H}}\right)+\left\{\begin{array}{l}
\lim _{\eta^{\prime} \rightarrow \infty}\left(-u^{\prime \prime}\right)+\pi \\
\lim _{\eta^{\prime} \rightarrow-\infty}\left(-u^{\prime \prime}\right) .
\end{array}\right.
$$

From Eq. (9), for $|\eta|>1$,

$$
\begin{aligned}
2 \sin ^{2}\left(-u^{\prime \prime}\right)= & {\left[\left(\eta^{\prime 2}+\eta^{\prime \prime 2}-1\right)+4 \eta^{\prime 2}\right]^{1 / 2} } \\
& -\left(\eta^{\prime 2}+\eta^{\prime \prime 2}-1\right)
\end{aligned}
$$

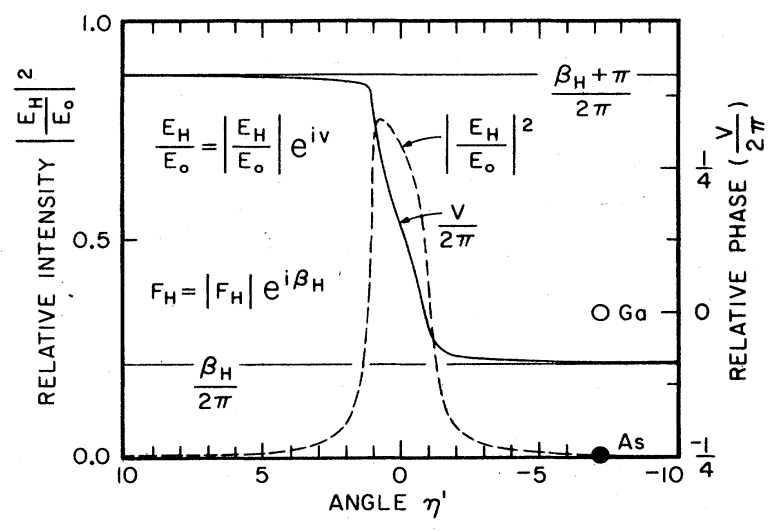

FIG. 4. Angular variation of the reflectivity and antinode position for $\mathrm{GaAs}(111)$ at $E_{i}=E_{k}^{\mathrm{As}}-6 \mathrm{eV}$. (See text for details.)

From Eq. (7)

$$
\begin{aligned}
\eta^{\prime \prime}=-\eta^{\prime} \tan \left(\frac{\beta_{H}+\beta_{\bar{H}}}{2}\right)+ & \frac{(1-b)}{2|b|^{1 / 2}|P|} \\
& \times \frac{F_{0}^{\prime \prime}}{\operatorname{Re}\left[\left(F_{H} F_{\bar{H}}\right)^{1 / 2}\right]},
\end{aligned}
$$

and

$$
\lim _{\left|\eta^{\prime}\right| \rightarrow \infty}\left(-u^{\prime \prime}\right)=\frac{\beta_{H}+\beta_{\bar{H}}}{2},
$$

Therefore, the asymptotic limits for the antinode position, as defined in Eq. (14) and shown in Fig. 4, are directly related to the phase of the structure factor as

$$
\frac{\Delta d_{0}}{d}=\frac{1}{2 \pi} \times\left\{\begin{array}{l}
\beta_{H}+\pi \text { for } \eta^{\prime} \rightarrow \infty \\
\beta_{H} \text { for } \eta^{\prime} \rightarrow-\infty
\end{array}\right.
$$

This asymptotic behavior can also be seen in Fig. 2 by comparing the phase angle $\beta_{H}$ of $F_{H}$ to the phase angle $v$ of $E_{H} / E_{0}$. For $P>0$, as $\eta^{\prime} \rightarrow \infty E_{H} / E_{0}$ goes to a counterphase alignment with respect to $F_{H}$ and for $\eta^{\prime} \rightarrow-\infty E_{H} / E_{0}$ goes to an in-phase alignment. The contour mapping of the complex field-amplitude ratio $E_{H} / E_{0}$ is constrained to lie tangent to $F_{H}$ at the origin of the complex plane. As $F_{H}$ rotates about this origin as a function of incident photon energy $E_{i}$, the $E_{H} / E_{0}$ contour rotates with respect to the fixed geometrical phase factors $s_{n}(H)$.

As described in Eq. (4), $\beta_{H}$ has an energy dependence due to anomalous dispersion. This is depicted in Figs. $3(\mathrm{~b})$ and $3(\mathrm{c})$ for the strong $F_{111}$ and weak $F_{200}$ zincblende structure factors of GaAs. For the simpler case of centrosymmetric diamond structure, all atoms in Fig. 1 would be replaced by $\mathrm{Ge}$ atoms. Consequently from Eq. (1), $F_{200}=0$, indicating a forbidden reflection. For $\mathrm{Ge}(111)$ with no absorption (i.e., $f^{\prime \prime}=0$ ), the energyindependent phase of the structure factor, as described in Eq. (4a), would be $\beta_{111}=-\pi / 4$. In this case, the (111) diffraction plane would coincide with the symmetry plane lying halfway between the two closely spaced (111) atom layers shown in Fig. 1. The inset of Fig. 2 illustrates this case in the complex plane. With absorption, the $\mathrm{Ge}(111)$ Bragg diffraction plane position at $\beta_{111} / 2 \pi$ is energy 
dependent and has a range corresponding to $4 \%$ of a Ge(111) $d$ spacing. As depicted in Figs. 3(b) and 3(c), this range is $8 \%$ for $\mathrm{GaAs}(111)$ and $100 \%$ for $\mathrm{GaAs}(200)$.

Referring to Eqs. (3) and (18), the two asymptotic positions for the standing-wave correspond to a counterphase and an in-phase condition with respect to the real part of $\rho_{H}(\mathbf{r})$. With our previous definition, the $(h k l)$ diffraction planes coincide with the maxima of $\operatorname{Re}\left[\rho_{H}(\mathbf{r})\right]$.

\section{GaAs(111) MEASUREMENT}

The first standing-wave fluorescence measurement of a noncentrosymmetric structure was performed by Trucano $^{10}$ on $\mathrm{GaP}(111)$, a structure which was subsequently also used for a standing-wave electron emission study by Takahashi and Kikuta. ${ }^{12}$ More recently, an XSW fluorescence measurement on GaAs(111) was made by Patel and Golovchenko. ${ }^{13}$ In both fluorescence studies, a conventional $\mathrm{x}$-ray source with a fixed energy was used and the fluorescence photons were detected by a solid-state detector. In the later measurement, ${ }^{13}$ the accuracy of the position determination was greatly improved by collecting the fluorescence signal at a glancing angle of $\alpha=0.4^{\circ}$ with respect to the (111) surface. This detector geometry, which is used for reducing the extinction effect, was also used in our present experimental setup (shown in Fig. 5). With the added features of synchrotron radiation, we were able to (1) selectively choose the incident photon energy, (2) essentially eliminate the influence of the Compton and thermal diffuse scattered signals [by collecting with the $\mathrm{Si}(\mathrm{Li})$ detector in the polarized direction of $\mathbf{E}_{0}$ ], and (3) increase the fluorescence and resonant Raman scattering (RRS) count rates by 2 orders of magnitude. All three of these features were necessary for observing the $K$-RRS signal, and for studying the energy dependence of $f^{\prime \prime}(H)$ and $\beta_{H}$. Referring to Eq. (4), this constitutes a measurement of $f^{\prime}(H)$ as well.

Dispersion correction $f^{\prime \prime}(0)$ was determined by using the setup shown in Fig. 5 (without diffraction from the GaAs sample) to measure the GaAs $K$ fluorescence and
$K$-RRS yields as a function of incident photon energy $E_{i}$. Since the secondary $\mathrm{x}$ rays were collected at a small glancing angle of $\alpha=1.6^{\circ}$, the variance in the effective thickness (which can mask the variance in the absorption cross section for the primary $x$ rays) was strongly reduced. This favorable reduction was especially true for the As $K$ fluorescence and As $K$-RRS photons, since the energies of these photons are just above the Ga $K$ edge. It was therefore possible to take the As $K$-edge yield spectrum shown in Fig. 6 as being proportional to $f_{\text {As }}^{\prime \prime}(0)$ plus an offset. The $f_{\mathrm{As}}^{\prime \prime}(0)$ scale was set by fixing $f_{\mathrm{As}}^{\prime \prime}(0)$ at -30 and +30 $\mathrm{eV}$ to values from Ref. 14 of 0.6 and 4.25 , respectively.

The arrows in Fig. 6 mark the energies at which the monochromator was set for a 10-min-long (111) x-ray standing-wave measurement of a symmetrically cut $(b=-1)$ GaAs (111) single crystal. Details of the instrumentation and analysis for this technique are given elsewhere. $6,9,20$ The measured angular intensities and theoretical fits for the standing-wave scan taken at $6 \mathrm{eV}$ below the As $K$ edge are shown in Fig. 7. The strong dip in the As $K$-RRS- $\beta$ yield at $30 \mu \mathrm{rad}$ above the geometrical Bragg angle $\theta_{B}$, is primarily due to the nodes of the standing wave passing through the As(111) atomic planes shown in Fig. 1. In reference to Fig. 2. a minimum $E$ field intensity at the As(111) atom planes corresponds to $E_{H} / E_{0}$ being in counterphase with $s_{\mathrm{As}}(111)$.

Based on Eq. (5), which describes the $E$-field intensity at the $n$th atom of a unit cell at a depth $z$ below the surface, and based on the attenuation $\mu_{\text {out }}^{n^{\prime \prime}}$ of a secondary photon from this depth which is escaping at a glancing angle $\alpha$ with respect to the surface, the angular dependence of the yield for a particular secondary process $n^{\prime \prime}$ from atom type $n^{\prime}$ in a single crystal can be expressed as

$$
\begin{aligned}
Y_{n^{\prime} n^{\prime \prime}}(\theta) \propto & 1+\left|\frac{E_{H}}{E_{0}}\right|^{2} \\
& \left.+2 f_{c} P\left|\frac{E_{H}}{E_{0}}\right| \cos \left(v+\Delta \beta_{H}-2 \pi \Phi_{c}\right)\right] Z(\theta) .
\end{aligned}
$$

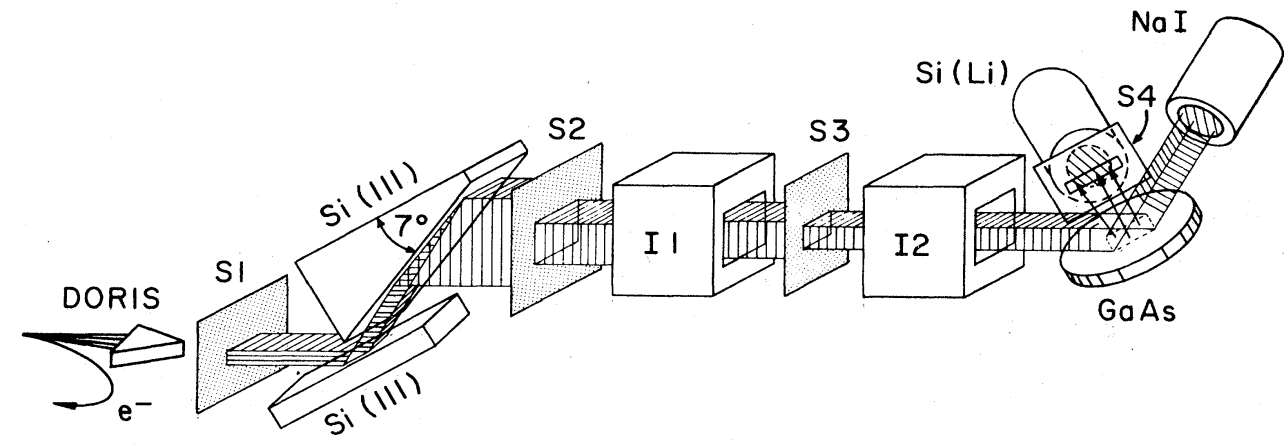

FIG. 5. Experimental setup at the ROEMO experimental station of HASYLAB, which was used for both the x-ray standing-wave and $f^{\prime \prime}$ measurements. The DORIS (Doppel-Ring Speicheranlage) storage ring was operating at an electron energy of $3.7 \mathrm{GeV}$ and a mean current of $60 \mathrm{~mA}$. The asymmetrical cut of the second monochromator (MC) crystal reduced both the energy and angular widths of emittance. (See $\Delta E_{\mathrm{MC}}$ and $\omega_{\mathrm{MC}}$ in Figs. 6 and 7.) The height and width of slit S3 was 0.4 and $0.7 \mathrm{~mm}$, respectively. Slit S4 limited the collection of secondary $\mathrm{x}$ rays in the $\mathrm{Si}(\mathrm{Li})$ detector to a glancing take-off angle of $\alpha=1.6^{\circ} \pm 0.5^{\circ}$ with respect to the surface of the GaAs(111) sample. 


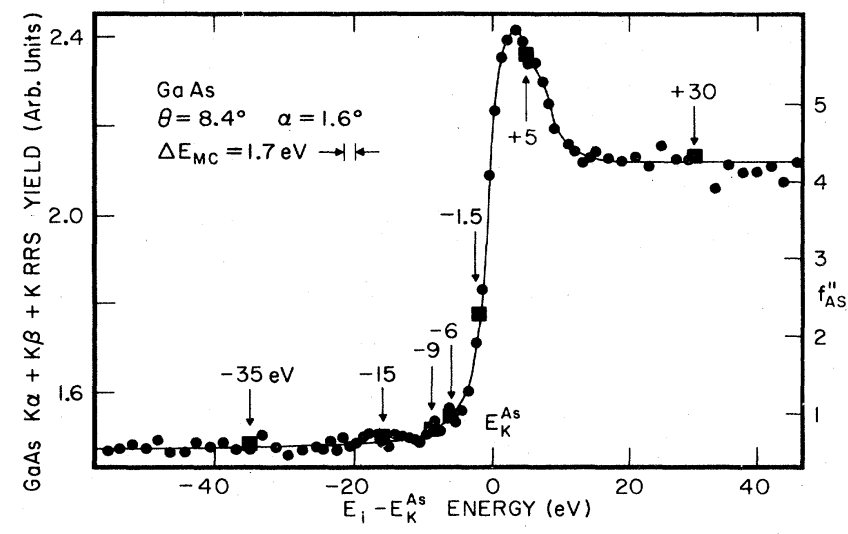

FIG. 6. Incident photon energy dependence of the $K$ fluorescence and $K$-RRS yield for GaAs near the As $K$ edge as measured by the $\mathrm{Si}(\mathrm{Li})$ detector relative to the ion chamber $\mathrm{I} 2$ shown in Fig. 5. (See text for details.)

For the experimental arrangement shown in Fig. 5, the incident synchrotron radiation is polarized perpendicular to both $\mathbf{K}_{0}$ and $\mathbf{K}_{H}$. Therefore, the polarization constant $P$ is equal to 1. The effective thickness used in Eq. (19) is

$$
Z_{n^{\prime \prime}}(\theta)=\left[\mu_{z}(\theta)+\mu_{\text {out }}^{n^{\prime \prime}} / \sin (\alpha)\right]^{-i},
$$

where the projected linear attenuation coefficients for the outgoing secondary photons in GaAs are

$$
\mu_{\mathrm{out}}^{\mathrm{Ga} K \alpha} / \sin \left(1.6^{\circ}\right)=0.862 \mu \mathrm{m}^{-1}
$$

and

$$
\mu_{\text {out }}^{\mathrm{As} K \beta} / \sin \left(1.6^{\circ}\right)=1.898 \mu \mathrm{m}^{-1}
$$

and the effective absorption coefficient ${ }^{2,9}$ for the incident radiation in the $E_{i}=E_{K}^{\mathrm{As}}-6 \mathrm{eV}, b=-1, \operatorname{GaAs}(111)$ Bragg reflection case is

$$
\mu_{z}\left(\eta^{\prime}= \pm 100\right)=0.329 \mu \mathrm{m}^{-1}
$$

and $\mu_{z}\left(\eta^{\prime}=0\right)=2.909 \mu \mathrm{m}^{-1}$. The coherent fraction used in Eq. (19) is defined as

$$
f_{c}=C \frac{f_{n^{\prime} n^{\prime \prime}}^{\prime \prime}(H)}{f_{n^{\prime} n^{\prime \prime}}^{\prime \prime}(0)} \frac{1}{N^{\prime}}\left|\sum_{n^{\prime}}^{N^{\prime}} s_{n^{\prime}}(H)\right| D_{n^{\prime}}(H),
$$

and the coherent position is defined as

$$
\Phi_{c}=(1 / 2 \pi) \arg \left(\sum_{n^{\prime}}^{N^{\prime}} s_{n^{\prime}}(H)\right) .
$$

Equation (22) is equivalent to the expression

$$
\sum_{n^{\prime}}^{N^{\prime}} s_{n^{\prime}}(H)=\left|\sum_{n^{\prime}}^{N^{\prime}} s_{n^{\prime}}(H)\right| \exp \left(2 \pi i \Phi_{c}\right) .
$$

The sums in Eqs. (21)-(23) are over the $N^{\prime}$ atoms of type $n^{\prime}$ in the unit cell. For $\mathrm{GaAs}(111) \Phi_{c}^{\mathrm{Ga}}=0$ and $\Phi_{c}^{\mathrm{As}}=-\frac{1}{4}$ in our chosen coordinate system. The commensurate fraction $C$ is equal to unity for a perfect crystal. In the dipole approximation for the photoeffect $f^{\prime \prime}(H) / f^{\prime \prime}(0)=1 .^{18} \mathrm{~Pa}$ rameter $\Delta \beta_{H}$ is the small difference between the standing-wave measured value $\beta_{H}^{\mathrm{XSW}}$ and the trial value of $\beta_{H}$ which was used for calculating $v$. Parameters $\Delta \beta_{H}$

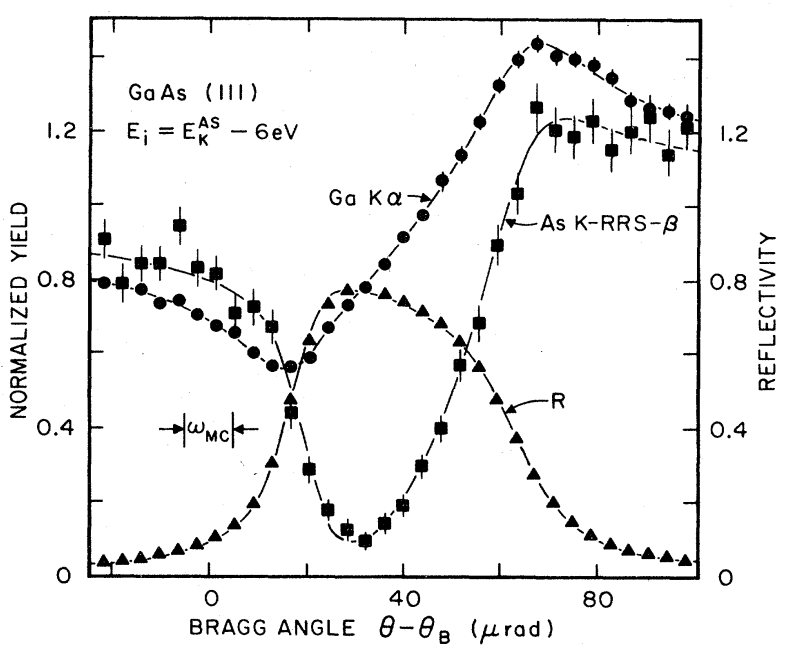

FIG. 7. Experimental data and theoretical curves for the $\mathrm{Ga}$ $K \alpha$ fluorescence, As $K$-RRS- $\beta$, and GaAs(111) reflectivity as a function of angle $\theta$ at $E_{i}=E_{K}^{\mathrm{As}}-6 \mathrm{eV}$. (See text for details.)

and $f_{c}$ are the unknowns to be determined by the $\chi^{2}$ fit of Eq. (19) to the data.

Such a fit is shown in Fig. 7 for $E_{i}=E_{K}^{\mathrm{As}}-6 \mathrm{eV}$. The measured phase from the As- $K$-RRS- $\beta$ yield was

$$
\beta_{111}^{\mathrm{XSW}}=2 \pi(-0.090 \pm 0.003) \text {. }
$$

This agrees with the predicted $\beta_{111}$ value shown in Fig. 3(b) for $E_{i}=E_{K}^{\mathrm{As}}-6 \mathrm{eV}$. For the Ga $K \alpha$ fluorescence yield (shown in Fig. 7)

$$
\beta_{111}^{\mathrm{XSW}}=2 \pi(-0.093 \pm 0.002) \text {. }
$$

Although this $\mathrm{Ga} K \alpha$ measurement of $\beta_{111}$ agrees with the above As $K$-RRS- $\beta$ measurement, the $\mathrm{Ga} K \alpha$ measurements for $E_{i}>E_{K}^{\mathrm{As}}-6 \mathrm{eV}$ systematically produced $\beta_{111}^{\mathrm{XSW}}$ values which were more negative than the As $K \alpha$ measured values. This discrepancy is due to a secondary effect in which some of the $\mathrm{Ga} K$ fluorescence is being induced by As $K$ fluorescence. For these energies $\Phi_{c}^{\mathrm{Ga}}$, as defined in Eq. (22), was not purely related to the interaction of the wave field with the atoms in the Ga(111) atomic planes. Therefore, the measured $\beta_{111}^{\mathrm{XSW}}$ values shown in Fig. 3(b) were determined by using the As $K \beta$ fluorescence signal for $E_{i}>E_{K}^{\mathrm{As}}-6 \mathrm{eV}$ and the $\mathrm{Ga} K \alpha$ fluorescence signal for $E_{K}^{\mathrm{Ga}}<E_{i}<E_{K}^{\mathrm{As}}-6 \mathrm{eV}$. The measured coherent fraction values $f_{c}$, corresponding to the above measured $\beta_{111}^{\mathrm{XSW}}$ values, were in a range between 0.95 and 0.99 with errors of \pm 0.01 . From Eq. (21), the expected value for an atom with one inequivalent position in a perfect crystal would be $f_{c}=D_{n^{\prime}}(111)=0.979$, if $f_{n^{\prime} n^{\prime \prime}}^{\prime \prime}(111)=f_{n^{\prime} n^{\prime \prime}}^{\prime \prime}(0)$. The measured $\beta_{111}^{\mathrm{XSW}}$ value at 10.07 $\mathrm{keV}$ is from our previous measurement ${ }^{9}$ with the lowresolution electron counter. ${ }^{21}$

The measured $\beta_{111}^{\mathrm{XSW}}$ values were used in Eq. (4a) to determine $f_{\text {As }}^{\prime}(111)$ in the vicinity of the As $K$ edge. In this analysis, $f_{\mathrm{Ga}}^{\prime}(111)$ and $f_{\mathrm{Ga}}^{\prime \prime}(111)$ were set at the values shown in Fig. 3(a) and $f_{A s}^{\prime \prime}(111)$ was set to the values shown in Fig. 6. The measured $f_{\text {As }}^{\prime}(111)$ values from this analysis are shown in Fig. 3(a) to be in good agreement with the $f_{\mathrm{As}}^{\prime}(0)$ curve ${ }^{14}$ determined from the $f_{\mathrm{As}}^{\prime \prime}(0)$ curve 
using the dispersion relation. In a similar fashion, $f_{\mathrm{As}}^{\prime}(111)$ was determined at 12,13 , and $15 \mathrm{keV}$, and $f_{\mathrm{Ga}}^{\prime}(111)$ was determined at $10.07 \mathrm{keV}$ and at $E_{K}^{\mathrm{Ga}}+24$ $\mathrm{eV}$.

\section{GaAs(200) MEASUREMENT}

The experimental setup shown in Fig. 5 was also used for the (200) XSW measurement of a symmetrically cut GaAs(200) single crystal. The GaAs(200) Bragg reflection rocking curve and the corresponding angular yield of the $\mathrm{Ga} K \alpha$ fluorescence for $E_{i}=E_{K}^{\mathrm{Ga}}+25 \mathrm{eV}$ are shown along with theoretical fits in the bottom of Fig. 8. At this energy, the effective emittance width from the asymmetric $\mathrm{Si}(111)$ monochromator crystal was $15.3 \mu \mathrm{rad}$ as compared to an acceptance width of $7.7 \mu \mathrm{rad}$ for the weak $\mathrm{GaAs}(200)$ reflection. Even with this rather large angular averaging, the movement of the standing wave with respect to the $\mathrm{Ga}(200)$ atomic planes is clearly seen by the modulation in the $\mathrm{Ga} K \alpha$ fluorescence yield. Due to anomalous dispersion, the phase of the $\mathrm{Ga} K \alpha$ modulations shown in Fig. 8 changes by approximately $\pi$ radians as the incident photon energy $E_{i}$ is increased from $E_{K}^{\mathrm{Ga}}+25 \mathrm{eV}$ to $E_{K}^{\mathrm{As}}-1.5 \mathrm{eV}$.

The GaAs(200) reflection is inherently weak, since the $\mathrm{Ga}(200)$ and the $\mathrm{As}(200)$ atom layers (as shown in Fig. 1) are separated by $\frac{1}{2} d_{200}$. This corresponds to the geometrical phase factors $s_{\mathrm{Ga}}(200)$ and $s_{\mathrm{As}}(200)$ being in counterphase. The energy dependence for the strength of this reflection, in terms of the ratio

$\left|F_{200}^{\prime}\right| / F_{0}^{\prime \prime}=\left|f_{\mathrm{Ga}}^{0}+f_{\mathrm{Ga}}^{\prime}-f_{\mathrm{As}}^{0}-f_{\mathrm{As}}^{\prime}\right| D /\left(f_{\mathrm{Ga}}^{\prime \prime}+f_{\mathrm{As}}^{\prime \prime}\right)$,

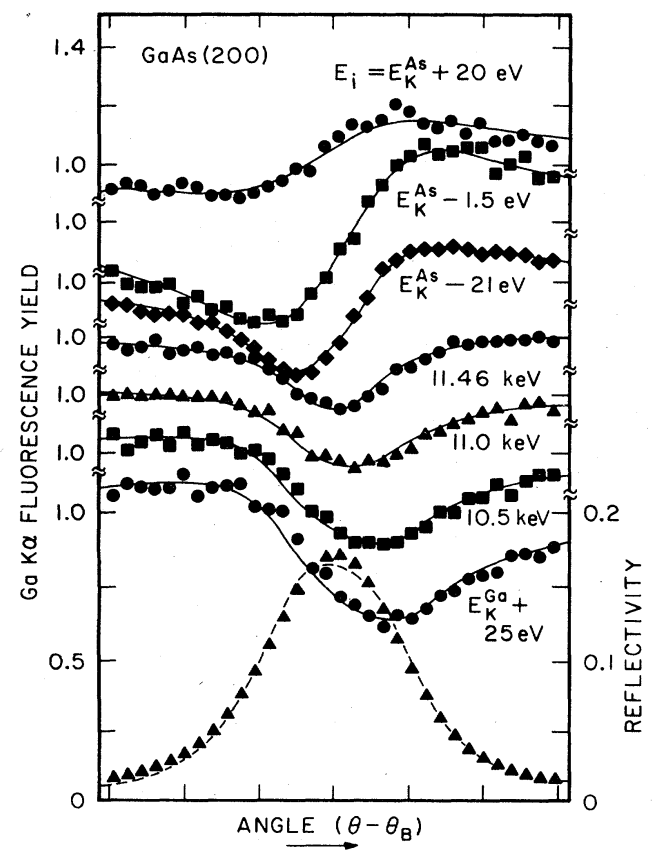

FIG. 8. Experimental data and theoretical curves for the $\mathrm{GaAs}(200)$ Bragg reflection and the $\mathrm{Ga} K \alpha$ fluorescence yield at $E_{i}=E_{K}^{\mathrm{Ga}}+25 \mathrm{eV}$ are shown at the bottom. Using the same vertical scale factor, subsequent $\mathrm{Ga} K \alpha$ yield curves are given vertical offsets of 0.2 . The angular range for each XSW scan was $\sim 50 \mu \mathrm{rad}$. (See text for details.) and the structure factor phase $\beta_{200}$ is described in Fig. 3 (c). In going from 8 to $15 \mathrm{keV}, F_{200}$ swings through all four quadrants of the complex plane. At $E_{i}=E_{K}^{\mathrm{Ga}}-5 \mathrm{eV}$, the reflection strength $\left|F_{200}^{\prime}\right| / F_{0}^{\prime \prime}$ is at a maximum and $F_{200}$ is in phase with $s_{\text {As }}(200)$. Therefore, at this energy the (200) Bragg diffraction planes at $\beta_{200} / 2 \pi$ coincide with the As $(200)$ atomic planes. From our previous definition of the Bragg diffraction plane, this means that the antinode of the standing wave moves inward from the $\mathrm{Ga}(200)$ atomic plane to the $\mathrm{As}(200)$ atomic plane as angle $\theta$ is increased through the $\mathrm{GaAs}(200)$ Bragg reflection. As shown in Fig. 3(c), the (200) Bragg diffraction plane (at $\beta_{200} / 2 \pi$ ) is slightly below the $\operatorname{As}(200)$ atomic layer for $E_{i}=E_{K}^{\mathrm{Ga}}+25 \mathrm{eV}$. This is in good agreement with the $\beta_{200}^{\mathrm{XSW}}$ value determined from the $\chi^{2}$ fit of Eq. (19) to the data shown in Fig. 8. In reference to Fig. 3(c), as $E_{i}$ is increased from $E_{K}^{\mathrm{Ga}}-5 \mathrm{eV}$ to $11.3 \mathrm{keV}$, the reflection strength decreases until it reaches zero at $11.3 \mathrm{keV}$ and the phase $\beta_{200}$ decreases by $\pi / 2$ radians. At $11.3 \mathrm{keV}$,

$$
F_{200}^{\prime}=4\left[f_{\mathrm{Ga}}^{0}(H)+f_{\mathrm{Ga}}^{\prime}(H)-f_{\mathrm{As}}^{0}(H)-f_{\mathrm{As}}^{\prime}(H)\right]=0
$$

is analogous to a $\mathrm{Ge}(200)$ forbidden reflection condition. As $E_{i}$ is increased from $11.3 \mathrm{keV}$ to $E_{K}^{\mathrm{As}}-2 \mathrm{eV}$, the reflection strength increases and the phase $\beta_{200}$ decreases by another $\pi / 2$ radians. The increased reflection strength is evidenced by the increase in the amplitudes of the modulations shown in Fig. 8. In going from the $\mathrm{Ga} K$ edge to the As $K$ edge, the phase of the structure factor has rotated with respect to $s_{\mathrm{Ga}}(200)$ from being in counterphase to being in-phase. Therefore, as shown in Fig. 8, the minimum in the Ga $K \alpha$ yield starts out on the high-angle side of the reflection at $E_{i}=E_{K}^{\mathrm{Ga}}+25 \mathrm{eV}$ and moves over until it reaches the low-angle side at $E_{i}=E_{K}^{\mathrm{As}}-1.5 \mathrm{eV}$.

As shown in Fig. 3(c), the measured $\beta_{200}^{\mathrm{XSW}}$ values agree with the values calculated from Eq. (4b).

\section{CONCLUSION}

We have demonstrated the use of an x-ray standingwave field for measuring the phase $\beta_{H}$ of the structure factor. By varying the incident photon energy, this physical parameter was shown to go through significant changes near the absorption edges. These changes were shown to be directly related to the anomalous behavior of the dispersion parameters. The combination of this phase measurement with the measured change in absorption, was used as a new approach for the direct determination of $f^{\prime}(H)$ and $f^{\prime \prime}(H)$ for atoms which appear in single crystals with a known structure. The present study has unambiguously demonstrated that the combination of reflectivity and emission yield measurements gives information about the amplitude and the phase of the structure factor. It is left as a future challenge to use this method for determining the positions of atoms in single crystals with unknown structures. It will also be of interest to apply this method to a case in which $f_{n^{\prime} n^{\prime \prime}}^{\prime \prime}(H)$ and $f_{n^{\prime} n^{\prime \prime}}^{\prime \prime}(H)$ are significantly different from $f_{n^{\prime} n^{\prime \prime}}^{\prime \prime}(0)$ and $f_{n^{\prime} n^{\prime \prime}}^{\prime \prime}(0)$, respectively.

Note added in proof. Professor Authier has informed us that he has independently been working on a similar calculation for the phase of $D_{H} / D_{0}$ for the absorbing 
case. Also related to this problem is a recent paper by $\mathbf{J}$. T. Hutton, G. T. Trammell, and J. P. Hannon [Phys. Rev. B 31, 6420 (1985)].

\section{ACKNOWLEDGMENTS}

One of the authors (M.B.) wishes to thank Professor B. W. Batterman and Dr. S. Durbin of Cornell University for several helpful discussions. This work is"a project of HASYLAB and was partly supported through the German Federal minister for Science and Technology (Bundesminister für Forschungs und Technologie). The final formulation in the theoretical part of this paper was also supported through CHESS by the National Science Foundation under Grant No. DMR-84-12465.
${ }^{1}$ M. v. Laue, Roentgenstrahl-Interferenzen (Akademische Verlagsgesellschaft, Frankfurt, 1960).

${ }^{2}$ B. W. Batterman and H. Cole, Rev. Mod. Phys. 36, 681 (1964).

${ }^{3}$ B. W. Batterman, Phys. Rev. 133, A759 (1964).

4J. A. Golovchenko, B. W. Batterman, and W. L. Brown, Phys. Rev. B 10, 4239 (1974).

5P. L. Cowan, J. A. Golovchenko, and M. F. Robbins, Phys. Rev. Lett. 44, 1680 (1980); M. J. Bedzyk, W. M. Gibson, and J. A. Golovchenko, J. Vac. Sci. Technol. 20, 634 (1982); J. A. Golovchenko, J. R. Patel, D. R. Kaplan, P. L. Cowan, and M. J. Bedzyk, Phys. Rev. Lett. 49, 560 (1982).

${ }^{6} \mathrm{G}$. Materlik and J. Zegenhagen, Phys. Lett. 104A, 47 (1984).

${ }^{7}$ M. J. Bedzyk and G. Materlik, Phys. Rev. B 31, 4110 (1985).

${ }^{8}$ N. Hertel, G. Materlik, and J. Zegenhagen, Z. Phys. B 58, 199 (1985).

${ }^{9}$ M. J. Bedzyk, G. Materlik, and M. V. Kovalchuk, Phys. Rev. B 30, 2453 (1984).

10P. Trucano, Phys. Rev. B 13, 2524 (1976).

${ }^{11}$ M. v. Laue, W. Fridrich, and P. Knipping, Munchener Sitzungsberichte 303 (1912); Ann. Phys. 41, 971 (1913).

12T. Takahashi and S. Kikuta, J. Phys. Soc. Jpn. 47, 620 (1979).

13J. R. Patel and J. A. Golovchenko, Phys. Rev. Lett. 50, 1858 (1983).

${ }^{14}$ T. Fukamachi, S. Hosoya, T. Kawamura, and M. Okunuki, Acta Cryst. A35, 828 (1979).

15International Tables for Crystallography (Kynoch, Birmingham, England, 1974), Vol. III.

${ }^{16} \mathrm{~L}$. Gerward, Nucl. Instrum. Methods 181, 11 (1981).

${ }^{17}$ P. Suortti, Phys. Status Solidi B 92, 259 (1979).

${ }^{18}$ H. Wagenfeld, Phys. Rev. 144, 216 (1966).

19J. H. Hubbell and I. Overbo, J. Phys. Chem. Ref. Data 8, 69
(1979).

${ }^{20}$ A. Krolzig, G. Materlik, and J. Zegenhagen, Nucl. Instrum. Methods 208, 613 (1983); A. Krolzig, G. Materlik, M. Swars, and J. Zegenhagen, Nucl. Instrum. Methods 219, 430 (1984).

${ }^{21}$ Using the present formulation of the diffraction plane position, Eqs. (8) and (9) of Ref. 9 should be rewritten as

$$
\frac{\Delta d_{0}}{d}=\lim _{\eta^{\prime} \rightarrow-\infty} \frac{v}{2 \pi}=\frac{1}{2 \pi} \arctan \left(\frac{F_{H}^{\prime \prime}}{F_{H}^{\prime}}\right)
$$

and

$$
\Delta_{111}=-\frac{1}{8}+\frac{1}{2 \pi} \arctan \left(\frac{f_{\mathrm{As}}^{0}+f_{\mathrm{As}}^{\prime}-f_{\mathrm{Ga}}^{\prime \prime}}{f_{\mathrm{Ga}}^{0}+f_{\mathrm{Ga}}^{\prime}+f_{\mathrm{As}}^{\prime \prime}}\right) .
$$

The $\Delta_{111}$ values, listed on Table I of Ref. 9, should be -0.011 , $-0.046,-0.011,-0.001$, and +0.006 , respectively, and the 15.1 and $10.07 \mathrm{keV}$ coherent position values of Table II of Ref. 9 should be given $\mathrm{a}-0.014$ and -0.004 offset, respectively. The measured coherent position of $\Phi_{c}^{\prime}=-0.014$ from a signal having a $G=0.43$ contributed fraction from $\mathrm{Ga}$ atoms is used in Eq. (14) of Ref. 9 to determine $\left(\beta_{111} / 2 \pi+\frac{1}{8}\right)=-\Delta_{111}=-0.008$ for $E_{i}=10.07 \mathrm{keV}$. The $G=0.43$ value was estimated from non- $K$ photoelectric cross sections. From the discussion of Ref. 9 it was presumed that a similar analysis could be applied to the high energy electron region of the 15.1-keV standing-wave scan. However, when applied, the produced value is in disagreement with our present study. This is due to errors in the electron spectrum background subtraction of Ref. 9 . 